\title{
Staphylococcus aureus carriage among health care workers at the regional hospital of Korca, Albania
}

\author{
Zhinzela Qyli \\ Nursing Department, Fan S Noli University, Korce, Albania
}

\begin{abstract}
Background. Health care workers (HCWs) are considered as one of the main sources of nosocomial infections. Staphylococcus aureus associated nosocomial infection remains a major health challenge. These infections continues to increase worldwide, particularly in poor countries where infection prevention is more difficult due to lack in hygiene and logistics.

Aim. This study aimed to make evident the prevalence of Staphylococcus aureus in nose among HCWs at the Regional Hospital of Korca Albania.

Methods. A total number of 102 swabs were collected from the HCWs of different departments of the hospital. Staphylococcus aureus identification was based on the classics methods of microbiology: culture characteristics, microscopy after Gram stain and biochemical tests.

Results. The swabs resulted positive for Staphylococcus aureus in 44 (43.1\%) samples.

Conclusions. The prevalence of Staphylococcus aureus in HCWs of the Regional Hospital of Korca is high. This is an indication of potential risk for nosocomial infections in the hospital.
\end{abstract}

Keywords: Staphylococcus aureus, nose, hospital

\section{BACKGROUND}

HCWs are considered as one of the main sources of nosocomial infections. Staphylococcus aureus associated nosocomial infection remains a major health challenge. These infections continue to increase worldwide, particularly in poor countries where infection prevention is more difficult due to lack in hygiene and logistics [1].

Staphylococcus aureus is the main microbial agent that cause nosocomial infections. This problem becomes more serious because of the increasing resistance of this pathogen to various antibiotic, which complicates treatment of Staphylococcus aureus infections. Studies have shown that nasal and throat carrier of Staphylococcus aureus have an important role in acquiring and transmitting infections with this pathogen [2-4].
HCWs in hospitals especially serve as reservoir of multiresistant strains of Staphylococcus aureus $[5,6]$. This is associated with high risk of developing morbidity and mortality especially in postoperative patients. Therefore, infection prevention remains the most important thing to do.

Is evident that in healthy people, can be distinguished three patterns of Staphylococcus carriers: about $20 \%$ of people are persistent carriers, $60 \%$ are intermittent carriers, and approximately $20 \%$ almost never carry Staphylococcus aureus [2].

\section{AIM}

This study aimed to make evident the prevalence of Staphylococcus aureus in nose among HCWs at the Regional Hospital of Korca Albania. 


\section{METHODS}

A total number of 102 swabs were collected from the HCWs of different departments of the hospital. 28 samples were collected from the surgery department, 14 samples from orthopedic department, 12 samples from the Intensive Care Unit, 18 samples from obstetric department, 20 samples from gynecology department and 10 samples from neonatology department. 38 samples were collected from the doctors and 64 samples from the nurses of the hospital. 52 samples were collected from male HCWs and 50 from female HCWS. The collected swabs were cultured in Agar blood and incubated in $37^{\circ} \mathrm{C}$ for $18-24$ hours. The next step was the microbial identification of the positive samples. Staphylococcus aureus identification was based in the methods of classic microbiology: colonies characteristic, microscopy after Gram stain and biochemical tests.

Staphylococcus aureus produces white 1-2 $\mathrm{mm}$ in diameter colonies or yellow to cream. Some strains are beta-haemolytic in the presence of oxygen. Colonies are easily emulsified and slightly raised. In microscopy after Gram stain were observed Gram-positive cocci in grape-like clusters [7].

The biochemical test used for the identification of Staphylococcus aureus were the catalase test which distinguished catalase positive bacteris (staphylococci) from catalase negative bacteria (streptococci) and the slide coagulase test to distinguish Staphylococcus aureus from the other staphylococci.

\section{Statistical analysis}

Chi-square analysis was used. The analysis was conducted using the SPSS version 20.0. Statistical significance was considered to be the value of $\mathrm{p} \leq 0.05$.

\section{RESULTS}

TABLE 1. Culture results of HCWs

\begin{tabular}{|l|l|}
\hline Culture results & No/percentage \\
\hline St. aureus & $44(43.1 \%)$ \\
\hline Other bacteria & $2(1.9 \%)$ \\
\hline Negative cultures & $56(54.9 \%)$ \\
\hline Total & $102(100 \%)$ \\
\hline
\end{tabular}

From the total of 102 cultures, 44 (43.1\%) resulted positive for Staphylococcus aureus, 2 (1.9\%) cultures resulted positive for other bacteria and $56(54.9 \%)$ cultures resulted negative. This correlation was statistically significant $\chi^{2}=21.3-\mathrm{p}<0.01$.

TABLE 2. Prevalence of Staphylococcus aureus in hospital departments

\begin{tabular}{|l|c|c|}
\hline Hospital yards & Staphylococcus aureus n/(\%) & Total $\mathbf{n}$ \\
\hline Surgery & $15(53.5 \%)$ & 28 \\
\hline Orthopedic & $6(42.8 \%)$ & 14 \\
\hline Intesnsive Care Unit & $2(16.6 \%)$ & 12 \\
\hline Obstetric & $10(55.5 \%)$ & 18 \\
\hline Gynecology & $9(45.0 \%)$ & 20 \\
\hline Neonatology & $2(20.0 \%)$ & 10 \\
\hline Total (n) & $44(43.1 \%)$ & 102 \\
\hline
\end{tabular}

The highest prevalence of Staphylococcus aureus resulted from the samples of obstetric department $(55.5 \%)$. The lowest prevalence resulted in the Intensive Care Unit (16.6\%). The prevalence of Staphylococcus aureus in orthopedic department was $42.8 \%$, in the obstetric department $55.5 \%$, in gynecology department $45.0 \%$ and in neonatology department $20.0 \%$.

TABLE 3. Prevalence of Staphylococcus aureus according to the category of HCWs

\begin{tabular}{|l|c|c|}
\hline Category & Staphylococcus aureus $\mathbf{n}(\%)$ & Total $\mathbf{n}$ \\
\hline Doctors & $13(34.2 \%)$ & 38 \\
\hline Nurses & $31(48.4 \%)$ & 64 \\
\hline Total $\mathbf{n}$ & $44(43.1 \%)$ & 102 \\
\hline
\end{tabular}

The prevalence of Staphylococcus aureus among doctors was $34.2 \%$ and among nurses was $48.4 \%$.

TABLE 4. Prevalence of Staphylococcus aureus according to sex of HCWs

\begin{tabular}{|l|c|c|}
\hline Sex & Staphylococcus aureus n (\%) & Total $\mathbf{n}$ \\
\hline Male & $25(48.0 \%)$ & 52 \\
\hline Female & $19(38.0 \%)$ & 50 \\
\hline Total $\mathbf{n}$ & $44(43.1 \%)$ & 102 \\
\hline
\end{tabular}

The prevalence of Staphylococcus aureus in male HCWs $(48.0 \%)$ was higher than in female HCWs $(38.0 \%)$.

\section{DISCUSSIONS}

The percentage of positive isolates from Staphylococcus aureus in nose of HCWs at the Regional Hospital of Korca is high (43.1\%). The high percentage of positive results indicate a potential risk for nosocomial infections, especially surgical site infections at the operating theatres.

In different studies authors have found that the prevalence of Staphylococcus aureus carriage among HCWs varies between $16.8 \%$ and $56.1 \%$ [8-10]. 
If we make a comparative analysis we can find studies with similar results in Saudi Arabia 40\% [11] and in Benghazi 47.5\% [12]. We can find also studies with contrast findings in Nigeria 50\% [9], Ghana $25.5 \%$ [13] and Nepal 15.7\% [14].

The highest prevalence of Staphylococcus aureus in the hospital resulted from the samples of obstetric department $(55.5 \%)$. This is a high prevalence considering the risk for infection transmission to mother and child in the maternity units. Contrast results were reported from other studies in Nepal 12.5\% [14] and in India $30.3 \%$ [15].

The lowest prevalence of positive samples resulted in the Intensive Care Unit (16.6\%). This result is similar with the findings of the study in Nepal $14.3 \%$ [14] and contrast with the results in Italy $38.1 \%$ [16].

Prevalence of Staphylococcus aureus isolates was higher from the samples of nurses (48.4\%) comparing to doctors. Contrast results are reported in India $22.86 \%$ [15], in Argentina 22\% [17] and $14.4 \%$ in Kuwait [18].

In this study, the prevalence of Staphylococcus aureus in the samples collected from doctors was 34.2\%. Similar findings are reported in Argentina 30\% [17]. Contrast findings are reported in India 25\% [15], Kuwait 21\% [18] and Saudi Arabia 53\% [19].
Prevalence of Staphylococcus aureus isolates was higher from the samples of male HCWs $(48.0 \%)$ comparing with female. These findings are similar with the studies in Saudi Arabia 48\% [11] and contrast with studies in Argentina 36\% [17] and Ethiopia 36.8\% [20].

Prevalence of positive samples in females was $38 \%$. Similar results are reported in Saudi Arabia $32.1 \%$ [11]. Contrast results are reported in Argentina $27.3 \%$ [17] and Ethiopia 21.3\% [20].

\section{CONCLUSIONS}

The percentage of Staphylococcus aureus among HCWs of the Regional Hospital of Korca was high. Nosocomial infections can be prevented, therefore measures should be taken for infection prevention especially in the departments where the percentage of positive isolates was higher and the risk for developing infection after surgery procedures is high. Some of the measures include: compilation and practical implementation of protocols used in hospitals, surveillance of infections and antibiotic resistance, use of protective masks and hand washing, avoiding touching one's nose during work.

Conflict of interest: none declared Financial support: none declared

\section{REFERENCES}

1. Revelas A. Healthcare-associated Infections: A Public Health Problem. Niger Med J. 2012;53(2):59-64.

2. Kluytmans $\mathrm{J}$, van Belkum $\mathrm{S}$, Verbrugh $\mathrm{H}$. Nasal carriage of Staphylococcus aureus: Epidemiology, underlying mechanisms, and associated risks. Clin Microbiol Rev. 1997;10(3):505-520.

3. Weinstein HJ. The Relation between the Nasal-StaphylococcalCarrier State and the Incidence of Postoperative Complications. N Engl J Med. 1959; 260:1303-1308.

4. Wertheim HFL, Melles DC, Vos MC, van Leeuwen W, van Belkum A, Verbrugh HA, Nouwen JL. The role of nasal carriage in Staphylococcus aureus infections. Lancet Infect Dis. 2005;5(12):751-62.

5. Panesso D, Planet PJ, Diaz L, Hugonnet JE, Tran TT, Narechania A, et al. Methicillin-susceptible, vancomycin-resistant Staphylococcus aureus, Brazil. Emerg. Infect. Dis. 2015;21(10):1844-1848.

6. Dancer SJ, Noble WC. Nasal, Axillary, and Perineal Carriage of Staphylococcus Aureus Among Women: Identification of Strains Producing Epidermolytic Toxin. Journal of Clinical Pathology 1991; 44(8):681-684.

7. Andoni Robert. Clinical bacteriology. Tirane, 2008.

8. Dan M, Moses Y, Poch F, Asherov J, Gutman R. Carriage of methicillin-resistant Staphylococcus aureus by non-hospitalized subjects in Israel. Infection. 1992;20(6):332-335.

9. Paul MO, Lamikanra A, Aderibigbe DA. Nasal carriers of coagulasepositive staphylococci in a Nigerian hospital community. Trans $R$ Soc Trop Med Hyg. 1982;76(3):319-323.

10. Mertz D, Frei R, Periat N, et al. Exclusive Staphylococcus aureus Throat Carriage. Arch Intern Med. 2009;169(2):172-178.

11. Al-Humaidan OS, El-Kersh TA, Al-Akeel RA. Risk factors of nasal carriage of Staphylococcus aureus and methicillin-resistant Staphylococcus aureus among health care staff in a teaching hospital in central Saudi Arabia. Saudi Med J. 2015;36(9):1084-1090.

12. Al-Abdli NE, Baiu SH. Nasal Carriage of Staphylococcus in Health Care Workers in Benghazi Hospitals. American Journal of Microbiological Research 2014;2(4):110-112.

13. Walana W, Bobzah BP, Kuugbee ED, et al. Staphylococcus aureus nasal carriage among healthcare workers, inpatients and caretakers in the Tamale Teaching Hospital, Ghana. Scientific African 2020; 8:e00325.

14. Shakya B, Shrestha S, Mitra T. Nasal carriage rate of methicillin resistant Staphylococcus aureus among at National Medical College Teaching Hospital, Birgunj, Nepal. Nepal Med Coll J. 2010;12(1):26-29.

15. Rongpharpi SR, Hazarika NK, Kalita H. The prevalence of nasal carriage of Staphylococcus aureus among healthcare workers at a tertiary care hospital in assam with special reference to MRSA. J Clin Diagn Res. 2013;7(2):257-260.

16. Pan A, Lorenzotti S, Ferrari L, Granata L, Signorini L, Carnevale G. Low rates of nasal colonization with methicillin-resistant Staphylococcus aureus among staff members of an Italian hospital. Infect Control Hosp Epidemiol. 2006 Feb;27(2):218-20.

17. Boncompain CA, Suárez CA, Morbidoni HR. Staphylococcus aureus nasal carriage in health care workers: First report from a major public hospital in Argentina. Revista Argentina de Microbiología. 2017;4 9(2):125-31. 
18. Dimitrov T, Udo EE, Grover S. Point surveillance of Staphylococcus aureus carriage among medical staff in Infectious Diseases Hospital, Kuwait. Med Princ Pract. 2003;12(3):139-144.

19. Ahmad S. Prevalence of Staphylococcus aureus colonization among healthcare workers at a specialist hospital in Saudi Arabia. Journal of Clinical and Diagnostic Research. 2010;(4):2438-41.
20. Shibabaw A, Abebe T, Mihret A. Nasal carriage rate of methicillin resistant Staphylococcus aureus among Dessie Referral Hospital Health Care Workers; Dessie, Northeast Ethiopia. Antimicrob Resist Infect Control. 2013;2(1):25. 